\title{
A NEW METHOD TO LOCATE FAULTS IN POWER NETWORKS BASED ON ELECTROMAGNETIC TIME REVERSAL
}

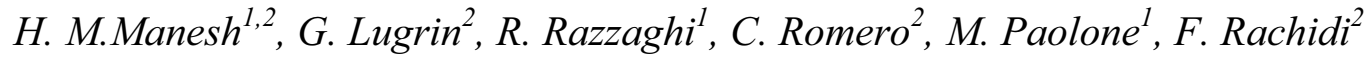 \\ École Polytechnique Fédérale de Lausanne (EPFL) \\ ${ }^{1}$ Distributed Electrical Systems Laboratory \\ ${ }^{2}$ Electromagnetic Compatibility Laboratory \\ Lausanne, Switzerland
}

\begin{abstract}
The paper presents a new method based on the Electromagnetic Time-Reversal (EMTR) for locating faults in power systems. The applicability of the EMTR to electromagnetic transients associated with traveling waves in transmission lines originated by the fault is theoretically demonstrated. A new fault location technique is then proposed and illustrated for a simple case of a singleconductor transmission line, for which the performance of the proposed technique in terms of location accuracy is discussed. The use of the EMTR technique appears to be particularly promising for locating faults in passive and active electrical distribution networks in view of their radial structure.
\end{abstract}

Index Terms - Electromagnetic time-reversal, fault location, power systems protection, fault transient, smart grids.

\section{INTRODUCTION}

Fault location functionality is an important on-line process required by power systems operation. It has a large influence on the security and quality of supply.

In transmission networks, this functionality is needed for the identification of the faulted line, and the adequate reconfiguration of the network to anticipate severe cascading consequences. In distribution networks, fault location is more associated to the quality of service in terms of duration of interruptions when permanent faults occur. Still with reference to distribution networks, the increasing diffusion of distributed generation calls for accurate and fast fault location procedures aimed at minimizing the network service restoration time and, consequently, minimizing the unsupplied power.

As summarized in [1], various procedures for fault location assessment have been proposed for both transmission and distribution power networks and two main categories can be identified: (i) analysis of pre- and post-fault voltage and current phasors (e.g. [2-4]) and, (ii) analysis of faultoriginated electromagnetic transients of currents and/or voltages, (i.e. travelling waves generated by the fault itself, e.g. [5-13]).

With particular reference to the case of distribution networks, typical methods used nowadays are based on the estimation of the post-fault impedance observed in measurement points usually located in primary substations. This estimation could provide useful information to locate the fault when compared with the line impedance with the hypothesis of having a fully passive power system. Indeed, the presence of other sources (e.g. associated with the increasing penetration of dispersed generation) can largely affect the accuracy of these procedures. For these reasons, procedures that belong to the second of the abovementioned categories may be less influenced by the presence of dispersed generation. This is because post-fault electromagnetic transients taking place within the first few milliseconds after the fault, are associated with the travelling waves originated by the fault itself and, therefore, are not influenced by the industrial-frequency power injections of distributed sources.

Within this context, this paper aims at investigating the possibility to apply to the problem of the fault location, the theory of Time-Reversal Process. This method was developed firstly in the field of acoustics [14-17] and was more recently applied to electromagnetics under the name Electromagnetic Time-Reversal (EMTR) (e.g. [18-21]). In what follows, we will use the EMTR acronym only.

The basic idea of the EMTR is to take advantage of the reversibility in time of the wave equation. This concept allows to 'time-reverse' the transients observed in specific observation points of the system to focalize the energy that has originated the transient at the source location.

The EMTR technique is mostly used in inhomogeneous medium to focus a wave into a specific point with a good accuracy and, also, to locate sources that have produced disturbances in a specific domain. It is particularly efficient when the system is limited in space [17].

The use of the EMTR technique will be discussed in the paper with reference to the case of transmission line equations and will be applied to locate sources of transients originated by faults. In the literature on the subject of fault location in wire networks, an approach based on the use of the so-called "matched-pulse reflectometry", that embed 
some of the properties of the EMTR, has been presented in [22].

The structure of the paper is as follows. Section 2 describes the basic aspects of the EMTR theory. Section 3 illustrates the main characteristics of electromagnetic transients originated by faults in power systems. In Section 4, we demonstrate the application of the EMTR to locate faults in transmission lines. The proposed algorithm is illustrated for a simple case of a single-conductor transmission line in Section 5. Finally, Section 6 concludes the paper with final remarks and future perspectives.

\section{BASIC CONCEPT OF THE EMTR TECHNIQUE}

In this Section, we examine the properties of the transmission line equations under time reversal.

The time-reversal operator corresponds to the change of the sign of the time, i.e. to the following transformation

$$
t \mapsto-t
$$

An equation is defined as 'time-reversal invariant' if it is invariant under the application of the time-reversal operator. The voltage wave equation for a single-conductor lossless transmission line reads

$$
\frac{\partial^{2} u(x, t)}{\partial x^{2}}-L^{\prime} C^{\prime} \frac{\partial^{2} u(x, t)}{\partial t^{2}}=0
$$

where $u(x, t)$ is the line voltage at position $x$ and time $t, L^{\prime}$ and $C^{\prime}$ are the line inductance and capacitance, respectively. Time reversing (2) reads:

$$
\frac{\partial^{2} u(x,-t)}{\partial x^{2}}-L^{\prime} C^{\prime} \frac{\partial^{2} u(x,-t)}{\partial t^{2}}=0
$$

Therefore, if $u(x, t)$ is a solution of the wave equation, then $u(x,-t)$ is also a solution. In other words, as described in [1517], the wave equation is invariant under a time-reversal transformation if there is no absorption during propagation in the medium, which in our case, is the transmission line waveguide. That is the reason why, in this paper, we restrict the application of EMTR to lossless lines.

Concerning the practical implementation of the EMTR, let us assume that we have recorded a transient signal within a time window $T, s(x, t), t \in[0, T]$, at a point $x$ of a transmission line. The application of the EMTR to $s(x, t)$ reads

$$
s(x, t) \mapsto s(x, T-t)
$$

In addition to time reversal, a time delay $T$ is added, so that the time remains positive for the duration of interest.

It is worth noting that, although the EMTR is defined in time domain, it can also be applied in frequency domain using the following equivalence:

$$
f(\vec{r},-t) \leftrightarrow \mathrm{F}^{*}(\vec{r}, \omega)
$$

where $\mathrm{F}(\vec{r}, \omega)$ is the Fourier transform of $f(\vec{r}, t)$ and * denotes the complex conjugate. Note that (5) is valid if $f(\vec{r}, t)$ is real.

\section{ELECTROMAGNETIC TRANSIENTS ASSOCIATED WITH FAULTS IN POWER SYSTEMS}

A fault event into a power system can be associated with an injection in the power system itself of a step-like wave triggered by the fault occurrence. The fault-generated wave travels along the lines of the network and gets reflected at the line extremities which are characterized by reflection coefficients whose values depend on the line surge impedance and the input impedances of the connected power components. In particular, the line extremities can be grouped into three categories, namely: line terminations with power transformers, junctions to other lines, and the fault location. For each of these boundary conditions the following assumptions can be reasonably made $[13,23]$ :

- extremities where a power transformer is connected can be assumed, for the traveling waves, as open circuits, and therefore the relevant reflection coefficient is close to +1 ; indeed fault-originated travelling waves are characterized by a spectrum with high-frequency components for which the input impedance of power transformers is generally dominated by a capacitive behavior [23];

- extremities that correspond to a junction between more than two lines are characterized by a negative reflection coefficient;

- fault location: the reflection coefficient of the extremity where the fault is occurring is close to -1 , as the fault impedance value can be assumed to be significantly lower than the line surge impedance.

With the above assumptions and for a given network topology, it is possible to determine a certain number $p$ of paths, each one delimited between two extremities. Fig 1 illustrates these paths for a simplified network topology composed by a main feeder and a lateral.

An observation point $m$ where voltage or current waveforms are measured will observe a superposition of travelling waves associated with the various paths. 


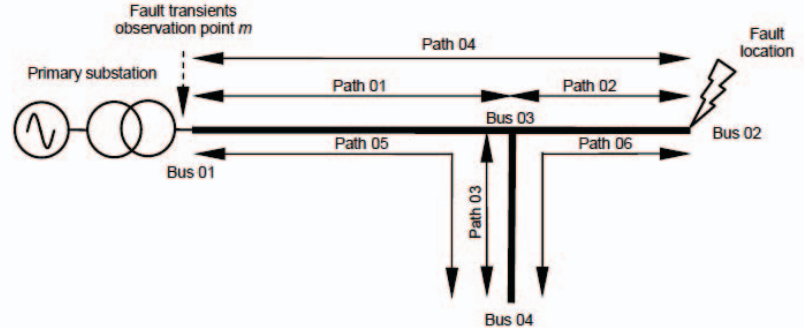

Fig. 1. Paths covered by travelling waves caused by a fault at Bus 02. Adapted from [24].

\section{APPLICATION OF THE EMTR METHOD TO FAULT LOCATION}

We present in this Section a new method based on the EMTR for fault location. As described in [14-20], one of the main hypotheses of the EMTR is that the topology of system needs to remain unchanged during the transient phenomenon of interest. Fault transients in power networks do not satisfy such a condition as the presence of the fault itself involves a change in the network topology when the fault occurs (i.e. at $t=t_{f}$ ). However, for reversed times until $t<T-t_{f}$, the EMTR is still applicable if a fault is considered at the correct position and, as we will see in this section, it allows to focalize the source of the fault.

In order to provide a more straightforward use of the EMTR, we will make reference to a single-conductor lossless transmission line (Fig. 2) of length $L$. The line parameters refer to a typical overhead transmission line. In particular, the surge impedance is in the order of a few hundred Ohms and the propagation speed, in view of the lossless representation, is the speed of the light, $c$.

We assume that at both line extremities power transformers are connected. Therefore, as discussed in Section 3, they are represented by means of high input impedances $\left(Z_{1}\right.$ and $Z_{2}$ in Fig. 1). The fault is placed at the coordinate $x_{f}$ and fault transient waveforms are assumed recorded at both ends of the line $\left(x_{1}=0, x_{2}=L,\right)$. As the line model is lossless, the damping of the transients is provided only by the fault impedance, if any, and the high terminal impedances $Z_{1}$ and $Z_{2}$.

Finally, as the analyzed fault transient lasts for only few ms, we assume that the pre-fault condition of the line is characterized by a constant value of voltage for $0 \leq x \leq L$.

\subsection{Frequency-domain expressions of voltages generated by the fault}

The aim of this sub-section is to analytically define the behavior of the line response after a fault. In order to express analytically the line response, the problem is formulated in frequency domain.
It is then necessary to specify the boundary conditions of the two line sections of Fig. 2, namely for $0 \leq x \leq x_{f}$ and $x_{f} \leq x \leq$ $L$. In view of the above assumptions, we can define reflection coefficients at $x=0$ (i=1 of Fig. 2) and $x=L$ ( $i=2$ of Fig. 2$)$ as

$$
\rho_{i}=\frac{\mathrm{Z}_{i}-\mathrm{Z}_{\mathrm{C}}}{\mathrm{Z}_{i}+\mathrm{Z}_{\mathrm{C}}} ; \quad i=1,2
$$

It is worth noting that $\rho_{i}$ in (6) could be assumed as frequency-independent parameters within the short observation time and in view of the high-frequency content of the fault transient.

Concerning boundary condition at the fault location, we assume to represent it by means of a voltage source $\mathrm{U}_{\mathrm{f}}(\omega)$ located at $x=x_{f}$.

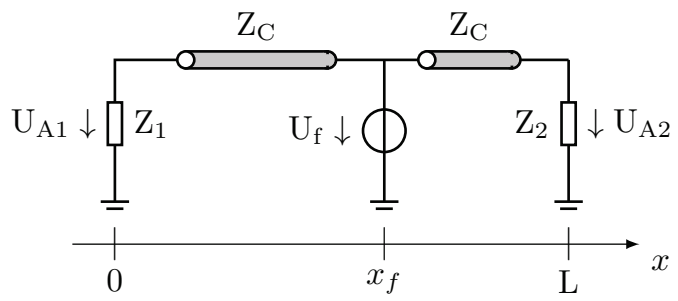

Fig. 2. Simplified representation of the post-fault line configuration.

As in the fault location we are assuming to place a voltage source, the relevant reflection coefficient $\rho_{f}=-1$. Additionally, in view of the lossless line assumption, the line propagation constant, $\gamma$, is purely imaginary, namely: $\gamma$ $=\mathrm{j} \beta$, with $\beta=\omega / c$. The expressions of the voltage observed in $x=0$ and $x=L$ in frequency domain read

$$
\begin{gathered}
\mathrm{U}_{\mathrm{A} 1}(\omega)=\mathrm{U}(0, \omega)=\frac{\left(1+\rho_{1}\right) \mathrm{e}^{-\gamma x_{f}}}{1+\rho_{1} \mathrm{e}^{-2 \gamma x_{f}}} \mathrm{U}_{\mathrm{f}}(\omega) \\
\mathrm{U}_{\mathrm{A} 2}(\omega)=\mathrm{U}(L, \omega)=\frac{\left(1+\rho_{2}\right) \mathrm{e}^{-\gamma\left(\mathrm{L}-x_{f}\right)}}{1+\rho_{2} \mathrm{e}^{-2 \gamma\left(\mathrm{L}-x_{f}\right)}} \mathrm{U}_{\mathrm{f}}(\omega)
\end{gathered}
$$

\subsection{EMTR applied to focus the fault location}

Equations (7) and (8) provide the frequency-domain expressions of fault-originated voltages in two observation points located at the line terminations. In agreement with the EMTR, we can replace these observation points with two sources each one imposing the time-reversed voltage fault transient, namely $\mathrm{U}_{\mathrm{A} 1}^{*}(\omega)$ and $\mathrm{U}_{\mathrm{A} 2}^{*}(\omega)$ where ${ }^{*}$ denotes the complex conjugate. Since the reflection coefficients $\rho_{1}$ and $\rho_{2}$ are almost equal to 1 , it is preferable to use the Norton equivalents as: 


$$
\begin{aligned}
& \mathrm{I}_{\mathrm{A} 1}^{*}(\omega)=\frac{\mathrm{U}_{\mathrm{A} 1}^{*}(\omega)}{\mathrm{Z}_{1}} \\
& \mathrm{I}_{\mathrm{A} 2}^{*}(\omega)=\frac{\mathrm{U}_{\mathrm{A} 1}^{*}(\omega)}{\mathrm{Z}_{2}}
\end{aligned}
$$

where $\mathrm{I}_{\mathrm{A} 1}^{*}(\omega)$ and $\mathrm{I}_{\mathrm{A} 2}^{*}(\omega)$ are the injected currents as shown on Fig. 3.

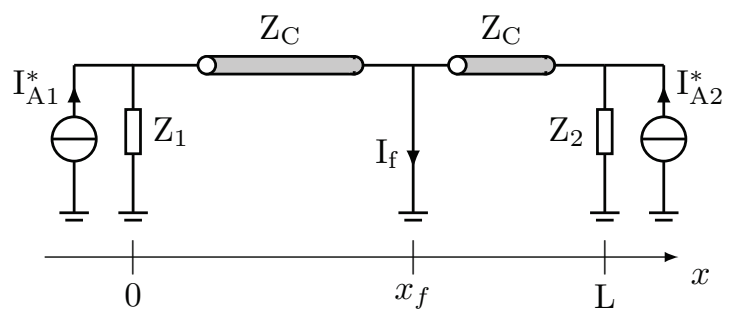

Fig. 3. Simplified representation of the EMTR applied to the single-line model of Fig. 2.

As the location of the fault is the unknown of the problem, we will place it at a generic distance $x_{f}^{\prime}$. The contributions in terms of currents at the unknown fault location $x_{f}^{\prime}$ coming from first and the second time-reversed sources $\mathrm{I}_{\mathrm{A} 1}^{*}(\omega)$ and $\mathrm{I}_{\mathrm{A} 2}^{*}(\omega)$, are given respectively by

$$
\begin{gathered}
\mathrm{I}_{\mathrm{f} 1}\left(x_{f}{ }_{f}, \omega\right)=\frac{\left(1+\rho_{1}\right) \mathrm{e}^{-\gamma x_{f}^{\prime}}}{1+\rho_{1} \mathrm{e}^{-2 \gamma x_{f}^{\prime}}} \mathrm{I}_{\mathrm{A} 1}^{*}(\omega) \\
\mathrm{I}_{\mathrm{f} 2}\left(x^{\prime}{ }_{f}, \omega\right)=\frac{\left(1+\rho_{2}\right) \mathrm{e}^{-\gamma\left(L-x_{f}\right)}}{1+\rho_{2} \mathrm{e}^{-2 \gamma\left(L-x_{f}^{\prime}\right)}} \mathrm{I}_{\mathrm{A} 2}^{*}(\omega)
\end{gathered}
$$

Introducing (7) and (8) into (11) and (12) reads:

$$
\begin{gathered}
\mathrm{I}_{\mathrm{f} 1}\left(x_{f}{ }_{f}, \omega\right)=\frac{\left(1+\rho_{1}\right)^{2} \mathrm{e}^{-\gamma\left(x_{f}^{\prime}-x_{f}\right)}}{\mathrm{Z}_{1}\left(1+\rho_{1} \mathrm{e}^{-2 \gamma x_{f}^{\prime}}\right)\left(1+\rho_{\mathrm{l}} \mathrm{e}^{+2 \gamma x_{f}}\right)} \mathrm{U}_{\mathrm{f}}^{*}(\omega) \\
\mathrm{I}_{\mathrm{f} 2}\left(x_{f}{ }_{f}, \omega\right)=\frac{\left(1+\rho_{2}\right)^{2} \mathrm{e}^{-\gamma\left(x_{f}-x_{f}^{\prime}\right)}}{\mathrm{Z}_{2}\left(1+\rho_{2} \mathrm{e}^{-2 \gamma\left(L-x_{f}^{\prime}\right)}\right)\left(1+\rho_{2} \mathrm{e}^{+2 \gamma\left(\mathrm{L}-x_{f}\right)}\right)} \mathrm{U}_{\mathrm{f}}^{*}(\omega)(14) .
\end{gathered}
$$

Therefore, we can obtain a closed-form expression of the total current at any location $x_{f}^{\prime}$ along the line

$$
\mathrm{I}_{\mathrm{f}}\left(x_{f}^{\prime}, \omega\right)=\mathrm{I}_{\mathrm{fl}}\left(x_{f}^{\prime}, \omega\right)+\mathrm{I}_{\mathrm{f} 2}\left(x_{f}^{\prime}, \omega\right)
$$

In what follows we will make use of (15) to show the applicability of the proposed method to locate the fault. Let us make reference to a line characterized by a total length
$L=10 \mathrm{~km}$ and let us assume a fault occurring at $x_{f}=8 \mathrm{~km}$. The line is characterized by terminal impedances $Z_{1}=Z_{2}=100 \mathrm{k} \Omega$ and, for the fault, we assume $U_{f}=1 / j \omega \mathrm{V} /(\mathrm{rad} / \mathrm{s})$. By varying $x_{f}^{\prime}$ from 0 to $L$, it is possible to compute the current along the line using (15). Fig. 4 shows the per-unit energy of $I_{f}$ within a spectrum between 0 to $2 \pi \cdot 10^{6} \mathrm{rad} / \mathrm{s}$. From Fig. 4 , it is clear that the energy of the signal is the largest at the fault location.

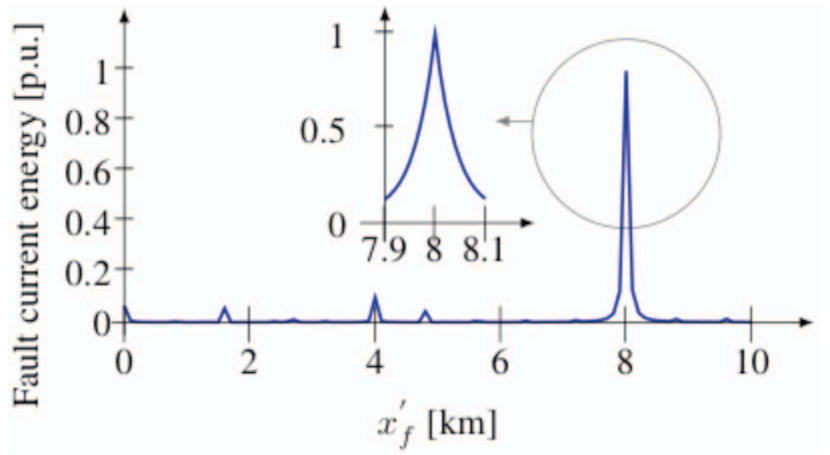

Fig. 4. Per-unit energy of the fault current as a function of the guessed fault location $x_{f}$. The real fault location is at $x_{f}=8 \mathrm{~km}$.

\section{APPLICATION EXAMPLES}

In this section, we present a numerical validation of the proposed technique. For this purpose, reference is made to the case of a single-conductor transmission line represented by means of a constant parameters model [24] implemented within the EMTP-RV simulation environment [25-27].

The adopted line parameters refer to a typical overhead transmission line and are reported in Table I.

Table. I. Adopted transmission line parameters.

\begin{tabular}{l|c}
\hline \hline \multicolumn{1}{c|}{ Line Parameter } & Value \\
\hline Length & $10 \mathrm{~km}$ \\
\hline Surge impedance $Z_{c}$ & $469 \Omega$ \\
\hline Per-unit length inductance & $1.56 \mu \mathrm{H} / \mathrm{m}$ \\
\hline Per-unit length capacitance & $7.10 \mathrm{pF} / \mathrm{m}$ \\
\hline Per-unit length resistance & $\approx 0$ \\
\hline \hline
\end{tabular}

The line is assumed to be terminated at its both ends on power transformers represented, as discussed in the previous sections, by high impedances, namely $100 \mathrm{k} \Omega$. The supply of the line is provided by an $\mathrm{AC}$ voltage source placed at $x=0$. A schematic representation of the system is shown in Fig. 5. 


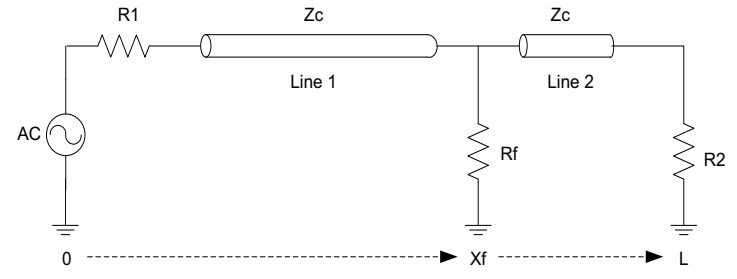

Fig. 5. Schematic representation of the transmission line implemented in the EMTP-RV simulation environment.

A fault is supposed to occur at $t=10 \mathrm{~ms}$ after the beginning of the simulation at $x_{f}=8 \mathrm{~km}$ with an impedance to ground equal to $1 \Omega$. The resulting transient voltages evaluated at both ends of the line are shown in Fig. 6 .
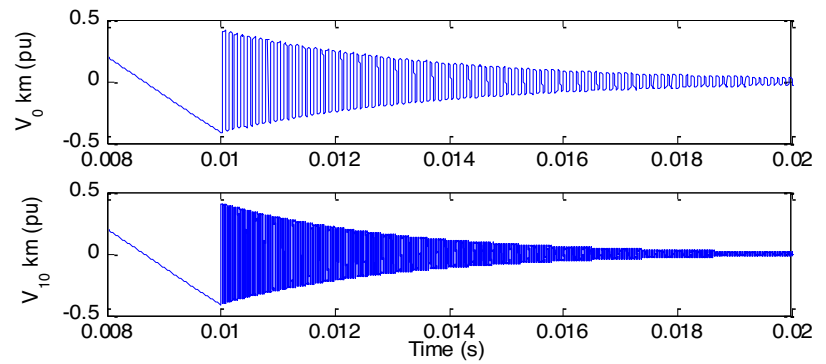

Fig. 6. Voltage transients recorded at both ends of the line of Fig. 5 and originated by a fault placed at $x_{f}=8 \mathrm{~km}, R_{f}=1 \Omega$.

In agreement with the EMTR, the recorded transients of Fig. 6 were reversed in time and injected back to the system (see Fig. 7). The guessed fault location is moved along the line assuming for the fault impedance a fixed value (as we will see this assumption does not influence the accuracy of the fault location). For each guessed fault location, the current flowing through the fault resistance is measured. In agreement with the discussion presented in Section 4, this current should exhibit a maximum in correspondence of the fault location. Fig. 8 shows the current for three guessed fault locations, namely $x_{f}^{\prime}=7,8$ and $9 \mathrm{~km}$. As expected, the highest current value is observed at the correct fault location, namely $8 \mathrm{~km}$. Additionally, it is worth noting that the fault current reaches its maximum value at the exact time-reversed fault inception time.

In order to verify the robustness of the proposed method against the assumed value of the fault impedance, Fig. 9 shows the peak values of the current flowing through the guessed fault location for three values of the fault resistance, namely 1,10 and $50 \Omega$. The current peak value is shown as a function of the guessed fault location, which is varied along the line with a step of $50 \mathrm{~m}$.

As it can be seen, the proposed method exhibits a clear robustness against the assumed fault impedance and, additionally, is characterized by an accuracy better than few tens of meters.
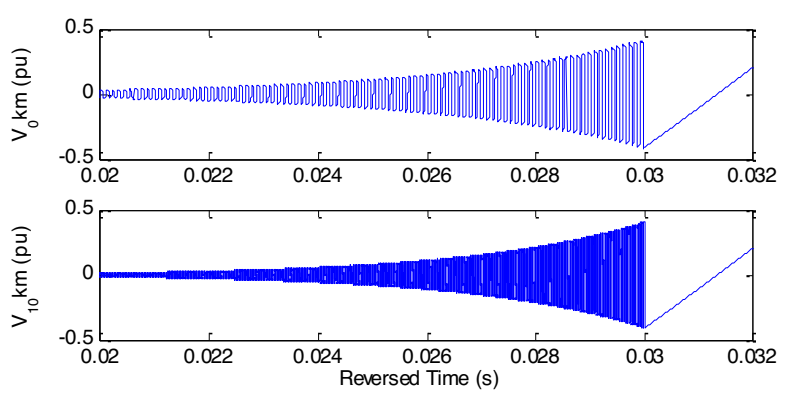

Fig. 7. Recorded transients in two measurement points at both ends of the line with the reversed time.

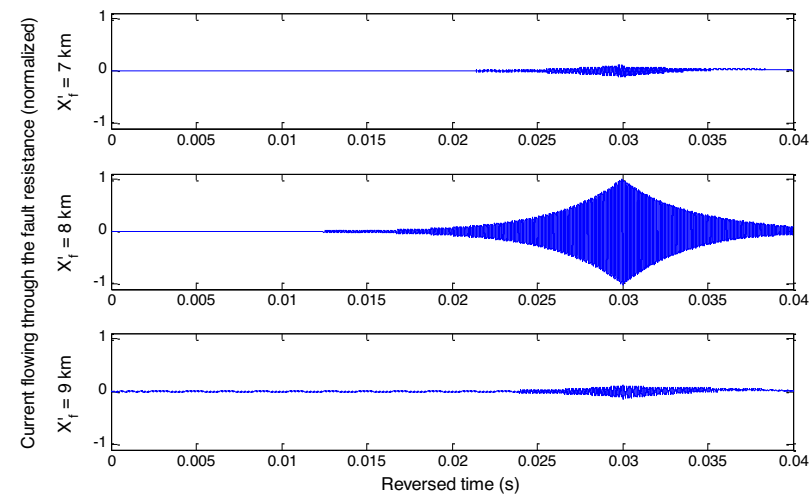

Fig. 8. Current flowing through the fault resistance for different guessed fault locations in the time reversal. Top: $7 \mathrm{~km}$, middle: 8 $\mathrm{km}$, bottom: $9 \mathrm{~km}$.

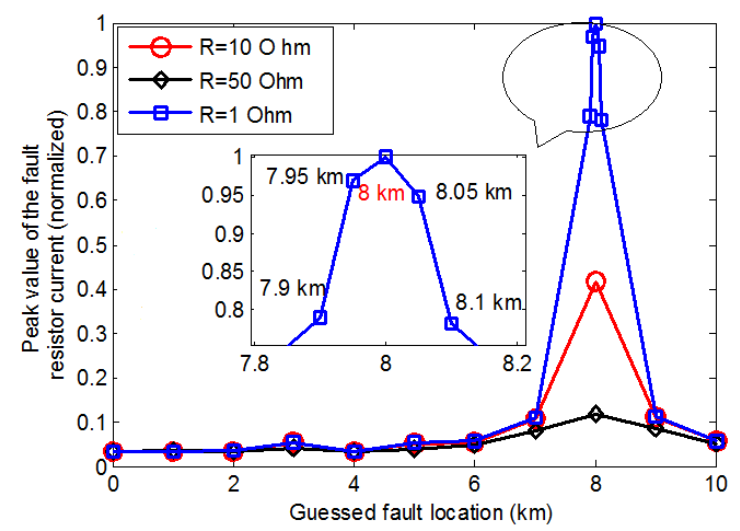

Fig. 9. The peak values of the fault resistor currents for different fault resisnace values.

\section{CONCLUSIONS}

We presented in this paper a new method to locate faults in power networks based on the use of the ElectroMagnetic Time Reversal (EMTR). By making reference to a simple transmission line configuration, the applicability of the EMTR to electromagnetic transients associated with traveling waves in transmission lines originated by a fault was theoretically demonstrated using closed-form analytical 
expressions in the frequency domain. The proposed method was also validated in time domain using numerical simulations obtained by means of the EMTP-RV simulation environment. The resulting fault location accuracy and robustness against uncertainties appear to be very promising for real applications. Further research is underway to extend the proposed method to take into account (i) multiconductor transmission lines, (ii) multi-terminal networks, and (iii) networks composed by overhead lines and cables.

\section{REFERENCES}

[1] IEEE Std C37.114, "IEEE guide for determining fault location on AC transmission and distribution lines", 2004.

[2] M. S. Sachdev and R. Agarwal, "A technique for estimating transmission line fault locations from digital impedance relay measurements" IEEE Trans. on PWRD, vol. 3, no. 1, pp. 121-129, Jan. 1988.

[3] K. Srinivasan and A. St.-Jacques, "A new fault location algorithm for radial transmission lines with loads," IEEE Trans. on PWRD, vol. 4, no. 3, pp. 1676-1682, Jul. 1989.

[4] A. A. Girgis, D. G. Hart, and W. L. Peterson, "A new fault location technique for two- and three-terminal lines," IEEE Trans. on PWRD, vol. 7, no. 1, pp. 98-107, Jan. 1992.

[5] G. B. Ancell and N. C. Pahalawatha, "Maximum likelihood estimation of fault location on transmission lines using travelling waves," IEEE Trans. on PWRD, vol. 9, no. 2, pp. 680-689, Apr. 1994.

[6] O. Chaari, M. Meunier, and F. Brouaye, "Wavelets: A new tool for resonant grounded power distribution systems relaying," IEEE Trans. on PWRD, vol. 11, no. 3, pp. 13011308, Jul. 1996

[7] F. H. Magnago and A. Abur, "Fault location using wavelets," IEEE Trans. on PWRD, vol. 13, no. 4, pp. 1475-1480, Oct. 1998.

[8] F. H. Magnago and A. Abur, "A new fault location technique for radial distribution systems based on high frequency signals," in Proc. IEEE-Power Eng. Soc. Summer Meeting, vol. 1, pp. 426-431, Jul. 18-22, 1999.

[9] D. W. P. Thomas, R. E. Batty, C. Christopoulos, and A. Wang, "A novel transmission-line voltage measuring method," IEEE Trans. Instrum. Meas., vol. 47, no. 5, pp. 1265-1270, Oct. 1998.

[10] Feng Yan, Zhiye Chen, Zhirui Liang, Yinghui Kong, Peng Li, "Fault location using wavelet packets", Proc. of Int. Conf. on Power System Technology, PowerCon 2002, Vol. 4, pp. 2575 - 2579, 13-17 Oct. 2002.

[11] A. Borghetti, S. Corsi, C. A. Nucci, M. Paolone, L. Peretto, and R. Tinarelli, "On the use of continuous-wavelet transform for fault location in distribution power networks," Elect. Power Energy Syst., vol. 28, pp. 608-617, 2006.

[12] A. Borghetti, M. Bosetti, M. Di Silvestro, C.A. Nucci and M. Paolone, "Continuous-Wavelet Transform for Fault Location in Distribution Power Networks: Definition of Mother Wavelets Inferred from Fault Originated Transients", IEEE Trans. on PWRS, vol. 23, No. 2, pp.380-388, may 2008.

[13] A. Borghetti, M. Bosetti, C.A. Nucci, M. Paolone, A. Abur, "Integrated Use of Time-Frequency Wavelet Decompositions for Fault Location in Distribution Networks: Theory and Experimental Validation", IEEE Trans. on PWRD, vol. 25, issue 4, Oct. 2010, pp. $3139-3146$.

[14] M. Fink, C. Prada, F. Wu and D. Cassereau, "Self focusing with time reversal mirror in inhomogeneous media", IEEE Ultrason. Symp., pp. 681-686, 1989.

[15] M. Fink, "Time reversal of ultrasonic fields. I. Basic principles", IEEE Trans. on Ultrasonics, Ferroelectrics and Frequency Control, vol. 39, issue 5, Sept. 1992, pp. $555-$ 566.

[16] F. Wu, J.-L. Thomas, M. Fink, "Time reversal of ultrasonic fields. II: Experimental results", IEEE Trans. on Ultrasonics, Ferroelectrics and Frequency Control, vol. 39, issue 5, Sept. 1992, pp: $567-578$.

[17] D. Cassereau, M. Fink, "Time-reversal of ultrasonic fields. III. Theory of the closed time-reversal cavity", IEEE Trans. on Ultrasonics, Ferroelectrics and Frequency Control, vol. 39, issue 5, Sept. 1992, pp: $579-592$.

[18] G. Lerosey, J. de Rosny, A. Tourin, A. Derode, G. Montaldo, M. Fink, Time Reversal of Electromagnetic Waves, Phys. Rev. Lett. 92, pp. 193904-193901-193903, 2004.

[19] D. Liu; G. Kang; L. Li; Y. Chen; S. Vasudevan, W. Joines, Q. Huo Liu, J. Krolik, L. Carin, "Electromagnetic timereversal imaging of a target in a cluttered environment", IEEE Trans. Antennas Propagat., vol. 53, pp. 3058-3066, 2005.

[20] H. Zhai, S. Sha, V. K. Shenoy, S. Jung, M. Lu, K. Min, S. Lee, D.S. Ha, "An Electronic Circuit System for TimeReversal of Ultra-Wideband Short Impulses Based on Frequency-Domain Approach", IEEE Transactions on Microwave Theory and Techniques,, vol. 58, issue: 1, pp. 74 $-86,2010$.

[21] N. Mora, F. Rachidi, M. Rubinstein, "Application of the Time Reversal of Electromagnetic Fields to Locate Lightning Discharges", Journal of Atmospheric Research, in press, 2012.

[22] A. Layane, A. Cozza and L. Pichon, "A Matched-Pulse Approach for Soft-Fault Detection in Complex Wire Networks", in press on IEEE Trans. on Instrumentation and Measurements, 2012.

[23] A. Greenwood, Electrical Transients in Power Systems, John Wiley and Sons, New YorkAJSA, 1991.

[24] A. Borghetti, M. Bosetti, C.A. Nucci, M. Paolone, A. Abur, "Fault location in active distribution networks by means of the continuous-wavelet analysis of fault-originated high frequency transients", Proc. of the Cigré General Session 2010, Aug. 22-27, 2010, Paris, France, paper C4-108.

[25] H. W. Dommel: "Digital computer solution of Electromagnetic Transiens in single and multiphase networks", IEEE Transactions, Vol. PAS-88, pages 388-399, April 1969

[26] J. Mahseredjian, S. Lefebvre and X.-D. Do, "A new method for time-domain modelling of nonlinear circuits in large linear networks", Proc. of 11th Power Systems Computation Conference PSCC, August 1993.

[27] J. Mahseredjian, S. Dennetière, L. Dubé, B. Khodabakhchian and L. Gérin-Lajoie: "On a new approach for the simulation of transients in power systems". Electric Power Systems Research, Volume 77, Issue 11, September 2007, pp. 15141520 . 